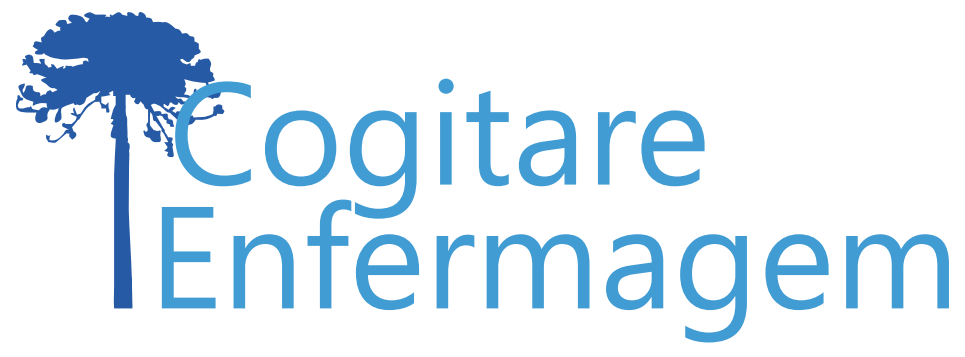

\title{
EVITABILIDADE DO ÓBITO INFANTIL E FETAL: INTERLOCUÇÃO ENTRE COMITÊ E ATENÇÃO PRIMÁRIA À SAÚDE
}

\author{
Alexandra Ferreira ${ }^{1}$, Selma Regina de Andrade², Andriela Backes Ruoff ${ }^{3}$, Laura Cavalcanti de \\ Farias Brehmer ${ }^{4}$, Ana Cristina Ammon Xavier ${ }^{5}$
}

\section{RESUMO}

Objetivo: evidenciar como ocorre a interlocução entre o Comitê de Prevenção do Óbito Materno, Infantil e Fetal e a Atenção Primária à Saúde no município de Florianópolis, Santa Catarina.

Método: estudo de caso único, com abordagem qualitativa, que teve como contexto o referido Comitê, justificado pelo caso decisivo, o Distrito Sanitário Norte. Na coleta de dados foram utilizadas quatro fontes de evidência e, na análise, a técnica de construção da explanação.

Resultados: evidenciou-se a implantação e organização do Grupo Técniço de Estudos sobre Mortalidade Materno-Infantil, como o agente interlocutor entre o Comitê e a atenção primária, que revisa condutas e processos de trabalho e propõe melhorias na assistência durante o pré-natal. Conclusão: as estratégias de gestão implementadas pelo Distrito Norte podem ser tomadas como exemplo para outros cenários, sobretudo para a promoção de uma interlocução efetiva com a atenção primária.

DESCRITORES: Gestão em saúde; Vigilância em saúde pública; Mortalidade infantil; Mortalidade fetal; Comitê de profissionais.

COMO REFERENCIAR ESTE ARTIGO:

Ferreira A, Andrade SR de, Ruoff AB, Brehmer LC de F, Xavier ACA. Evitabilidade do óbito infantil e fetal: interlocução entre comitê e atenção primária à saúde. Cogitare enferm. [Internet]. 2019 [acesso em "colocar data de acesso, dia, mês abreviado e ano"]; 24. Disponível em: http://dx.doi.org/10.5380/ce.v24i0.56649.

Este obra está licenciado com uma Licença Creative Commons Atribuição 4.0 Internacional.

${ }^{1}$ Enfermeira. Universidade Federal de Santa Catarina. Florianópolis, SC, Brasil. $\bigcirc$

${ }^{2}$ Enfermeira. Doutora em Enfermagem. Docente de Enfermagem da Universidade Federal de Santa Catarina. Florianópolis, SC, Brasil.

${ }^{3}$ Enfermeira. Doutoranda em Enfermagem. Universidade Federal de Santa Catarina. Florianópolis, SC, Brasil. $($ )

${ }^{4}$ Enfermeira. Doutora em Enfermagem. Docente de Enfermagem da Universidade Federal de Santa Catarina. Florianópolis, SC, Brasil.

${ }^{5}$ Enfermeira. Universidade Federal de Santa Catarina. Florianópolis, SC, Brasil. $\bigcirc$ 


\title{
AVOIDANCE OF INFANT AND FETAL DEATH: INTERLOCUTION BETWEEN THE COMMITTEE AND PRIMARY HEALTH CARE
}

\begin{abstract}
Objective: to investigate how the interlocution between the Committee for the Prevention of Maternal, Infant and Fetal Death and Primary Health Care takes place in the municipality of Florianópolis, Santa Catarina.

Method: a single case study, with a qualitative approach, which had the Committee mentioned as the context, justified by the decisive case of the Northern Health District. ,Four sources of evidence were used in the data collection and the explanatory construction technique in the analysis,.

Results: the implantation and organization of the Mother and Infant Mortality Study Technical Group was highlighted, this being the interlocutor agent between the Committee and primary care, which reviews behaviors and work processes and proposes improvements in prenatal care.

Conclusion: the management strategies implemented by the Northern District can be taken as an example for other scenarios, especially for the promotion of an effective interlocution with primary care.
\end{abstract}

DESCRIPTORS: Health management; Public health surveillance; Child mortality; Fetal mortality; Committee of professionals.

\section{EVITABILIDAD DEL ÓBITO INFANTIL Y FETAL: DIÁLOGO ENTRE COMITÉ Y ATENCIÓN BÁSICA A LA SALUD}

\section{RESUMEN:}

Objetivo: evidenciar cómo ocurre el diálogo entre el Comité de Prevención del Óbito Materno, Infantil y Fetal y la Atención Básica a la Salud en el municipio de Florianópolis, Santa Catarina. Método: estudio de caso único, con abordaje cualitativo, que tuvo como contexto el antedicho Comité, justificado por el caso decisivo, el Distrito Sanitario Norte. En la obtención de datos se utilizaron cuatro fuentes de evidencia y, en el análisis, la técnica de construcción de la explanación.

Resultados: se evidenciaron la implantación y la organización del Grupo Técnico de Estudios sobre Mortalidad Materno Infantil, como el agente de diálogo entre el Comité y la atención básica, que revisa conductas y procesos de trabajo y propone mejorías en la asistencia durante el prenatal.

Conclusión: las estrategias de gestión implementadas por el Distrito Norte pueden ser ejemplo para otros escenarios, sobre todo para la promoción de una interlocución efectiva con la atención básica.

DESCRIPTORES: Gestión en salud; Vigilancia en salud pública; Mortalidad infantil; Mortalidad fetal; Comité de profesionales. 
Utilizada mundialmente como um indicador sensível da qualidade de vida da população, a taxa de mortalidade infantil reflete as condições de vida da sociedade, por abranger diversos determinantes sociais do processo saúde-doença, como os fatores biológicos, ambientais, assistenciais e socioeconômicos. Compreende o número de óbitos de menores de um ano de idade por mil nascidos vivos, na população residente em determinado espaço geográfico, no ano considerado(1).

A redução da taxa de mortalidade infantil é um dos desafios persistentes dos serviços de saúde, com destaque nos Objetivos de Desenvolvimento Sustentável da Organização das Nações Unidas. É constituinte do Objetivo 3 - assegurar uma vida saudável e promover o bem-estar para todos, em todas as idades, que prevê, até 2030, acabar com as mortes evitáveis de recém-nascidos e crianças menores de 5 anos, além de reduzir a mortalidade neonatal para pelo menos até 12 por 1.000 nascidos vivos e a mortalidade de crianças menores de 5 anos para pelo menos até 25 por 1.000 nascidos vivos ${ }^{(2)}$.

Em 2016, 67,3\% ( $n=24.423)$ dos óbitos infantis e fetais no Brasil foram considerados evitáveis, ou seja, se fosse garantido, em tempo oportuno, acesso à assistência qualificada e resolutiva, tais óbitos não ocorreriam. Óbitos com potencial de prevenção são eventossentinela; sua ocorrência inicia uma investigação para compreender os fatores que levaram a esse desfecho, já que possivelmente sua ocorrência reflete falhas na atenção à saúde e indicam a necessidade de aprimoramento nos setores envolvidos no atendimento desse grupo populacional(3).

Em relação às classificações de evitabilidade, o componente com maior índice no cenário nacional foi a classificação "redutível por adequada atenção à gestação"(4-6). Este dado revela a existência de fragilidades na assistência realizada no pré-natal, indicando a necessidade de estratégias gerenciais para a redução dos óbitos evitáveis no âmbito da Atenção Primária à Saúde (APS) ${ }^{(7)}$.

Uma das estratégias para a redução da mortalidade infantil é a constituição de Comitê de Prevenção do Óbito Infantil e Fetal (CPOIF). Cada CPOIF é composto por uma equipe multiprofissional, com representantes de diversas instituições ligadas à saúde da criança, desde a atenção hospitalar à atenção primária. Propõe-se a dar visibilidade, monitorar e acompanhar os óbitos infantis e fétais, definindo estratégias e medidas de intervenção junto aos serviços ${ }^{(8)}$.

Com isto, a atuação do CPOIF tem papel significativo para a análise detalhada dos óbitos infantis e fetais, expondo as situações que necessitam de intervenção. É de sua atribuição analisar e discutir os óbitos com os profissionais envolvidos em todos os níveis de assistência ao binômio mãe-bebê, bem como elaborar as recomendações para que casos similares não aconteçam. Tais recomendações devem ser encaminhadas aos gestores das instituições de saúde como parte do trabalho da vigilância de óbitos e de reorganização dos serviços, com a finalidade de discutir as circunstâncias associadas aos óbitos e qualificar a assistência(9).

A devolução das recomendações às instituições de saúde requer mecanismos de interlocução entre gestores locais para efetivação dessa ação. Em Florianópolis-SC, o CPOIF possui o componente materno, sendo instituído como Comitê de Prevenção do Óbito Materno, Infantil e Fetal (CPOMIF). Um mecanismo de interlocução entre o CPOMIF e a APS em Florianópolis recebeu destaque com a criação de Grupo Técnico, em um Distrito Sanitário, como uma modalidade viável de efetivar as recomendações às unidades de saúde que atendem ao território definido.

Com base no exposto, este estudo adotou as seguintes proposições teóricas: (1) a maior parte dos óbitos classificados como evitáveis possuem relação com o pré-natal, cujo contexto predominante relaciona-se à APS; e (2) o CPOMIF tem a atribuição de promover a interlocução com a APS, com o intuito de reduzir o quantitativo de óbitos infantis e 
fetais evitáveis. Estas proposições dão suporte ao objetivo de evidenciar como ocorre a interlocução entre o CPOMIF e a APS em um Distrito Sanitário de Florianópolis.

\section{MÉTODO}

Estudo de caso único, do tipo decisivo, com abordagem qualitativa, explanatória e descritiva. Este tipo de estudo é recomendado quando, na seleção do caso, há um conjunto claro de circunstâncias que permite relacioná-las às proposições teóricas de interesse ${ }^{(10)}$. Neste estudo, no contexto do CPOMIF, o caso foi o Distrito Sanitário Norte (DSN), selecionado por ser o único no município com um Grupo Técnico de Estudos sobre Mortalidade Materno-Infantil (GTEM) implantado, que efetiva a interlocução das recomendações do CPOMIF à APS. Convém esclarecer que no CPOMIF os profissionais representantes dos cinco Distritos Sanitários (Centro, Continente, Norte, Leste e Sul) realizam a vigilância do óbito infantil, investigando-o, quando de sua ocorrência, nos contextos hospitalar, ambulatorial e domiciliar. Contudo, somente o Norte criou uma estrutura para atuar junto à APS, tornando-o decisivo para efetivação da interlocução.

A coleta dos dados se deu por quatro fontes de evidências: pesquisa documental, observação direta participante, observação direta não participante e entrevista focada com informante-chave. Os documentos utilizados na pesquisa documental foram as atas das reuniões ordinárias e extraordinárias do CPOMIF dos últimos três anos (2014 a 2016), totalizando 36 atas. A observação direta participante aconteceu nos anos de 2015 e 2016, por meio da experiência de extensão universitária realizada por uma das autoras junto à Coordenação de Saúde da Criança da Secretaria Municipal de Saúde, registrada em portfólios mensais das atividades e em quatro relatórios de acompanhamento das ações desenvolvidas.

A observação não participante ocorreu em duas reuniões do CPOMIF, nos meses de setembro e dezembro de 2016. Essa etapa contou com roteiro estruturado para nortear a observação de acordo com o objetivo do estudo. A entrevista focada com informantechave seguiu roteiro previamente elaborado, com perguntas abertas, sendo gravada e posteriormente transcrita. $O$ informante-chave desta pesquisa foi escolhido por atuar concomitantemente no CPOMIF, no DSN e no GTEM, a fim de compartilhar e buscar compreender o processo de trabalho que o torna um distrito sanitário diferenciado e único.

Para a análise dos dados, utilizaram-se as estratégias analíticas de orientação por proposições teóricas e de desenvolvimento da descrição do caso. Com elas, aplicouse a técnica de construção da explanação, formulando uma explicação para o caso. A organização e a sistematização dos dados para a análise foram feitas com o apoio do programa MaxQDA®plus.

O estudo seguiu as determinações da Resolução n 466/2012 do Conselho Nacional de Saúde e foi aprovado pelo Comitê de Ética em Pesquisa com Seres Humanos da Universidade Federal de Santa Catarina (CEP/UFSC), com o parecer $n^{\circ} 1.556 .889$.

\section{RESULTADOS}

Os resultados foram organizados em dois tópicos. Inicialmente, descreve-se o processo e dinâmica de trabalho do DSN para a vigilância do óbito infantil naquele território. Em seguida, faz-se a explanação do mecanismo de interlocução que o DSN promove entre o CPOMIF e a APS por meio do GTEM.

\section{O Caso Decisivo: Distrito Sanitário Norte, Florianópolis}


O DSN é constituído por onze Centros de Saúde, uma policlínica e uma Unidade de Pronto Atendimento, tendo sua sede administrativa anexa a esta última.

Em relação à mortalidade infantil, os profissionais da Vigilância Epidemiológica dos Distritos Sanitários, possuem representação no CPOMIF. Os casos são divididos conforme o território, sendo de responsabilidade da Vigilância Epidemiológica de cada distrito realizar a investigação do óbito materno, infantil ou fetal.

O processo de vigilância se inicia com a Declaração de Óbito (DO), seguindo com a investigação ambulatorial por meio do prontuário eletrônico da mulher, analisando toda a assistência no período gravídico. Se óbito infantil, coleta-se os dados adicionais do prontuário da criança.

Os profissionais da vigilância realizam a entrevista domiciliar, reconstruindo com a família os fatos que levaram ao óbito e registrando a percepção dos familiares sobre o desfecho. Executam, também, a investigação hospitalar na maternidade onde ocorreu o parto e na instituição que atendeu a criança até a constatação do óbito. O processo da investigação finda com a reconstrução da história em um resumo, ressaltando os pontos mais importantes do caso.

Após a realização do resumo, os óbitos são levados para discussão com a equipe multidisciplinar do CPOMIF, onde são analisados, elaborando os apontamentos e recomendações a todos os níveis de atenção para evitar casos similares.

O DSN, com o objetivo de ter um trabalho sistematizado que propicie a interlocução com a APS, implantou o GTEM. A necessidade da formação deste GTEM vem da percepção da limitação do CPOMIF quanto à alteração de processos de trabalho, e do interesse do DSN em reduzir a taxa de mortalidade infantil, realizando um trabalho mais próximo com as unidades de saúde do seu território.

A diferença entre Comitê e GTEM se dá na alteração do processo de trabalho. $O$ Comitê não delibera para alterar processos, ele apenas recomenda mudanças. Por sua vez, o GTEM, ao possuir em sua composição o gerente do Distrito, consegue alterar os processos de trabalho de maneira emergencial e resolutiva. Então, o GTEM do DSN possui caráter interventivo e resolutivo, agindo na sua área de abrangência, recomendando e agindo apenas para as instituições que compõe seu quadro formativo.

Nós queríamos alguma coisa pra agir [...], eu tenho que mudar esse processo de trabalho agora, eu não posso recomendar "vocês devem...", não, tem que mudar hoje, tem que intervir mesmo no processo de trabalho. [...] Então, a gente resolveu reunir esse grupo de pessoas com o objetivo de intervir. (Informante-chave)

A dinâmica das reuniões do GTEM é similar às do CPOMIF. Trata-se de um grupo multiprofissional e multidisciplinar, com reuniões mensais, sendo composto pelas apoiadoras da atenção primária, o gerente do DSN, as enfermeiras responsáveis pela vigilância do óbito, médico de família, pediatra e ginecologista obstetra. Apresenta-se o resumo do caso, as fragilidades e potencialidades, pontuam-se as situaçôes levantadas propondo as devidas recomendações e avalia-se o óbito, definindo-o como evitável, inevitável ou inconclusivo. Com a realização deste trabalho pelo GTEM, aponta-se a potencialidade de o caso ir mais maduro e detalhado ao Comitê, uma vez discutido e avaliado, as situações já foram apontadas e recomendações indicadas.

Anualmente acontece o seminário do DSN, onde são apresentados os resultados dos indicadores pactuados pelos gestores. Neste seminário, expõem-se os dados de mortalidade infantil, de forma a possibilitar que todos os profissionais do distrito tenham a visão da realidade do DSN, mesmo aqueles Centros de Saúde que não possuem óbitos.

Em relação às dificuldades encontradas, destaca-se a falta de profissionais e o fato de que, por ser profissional da rede, afasta-se o profissional da assistência durante o período 
de realização da reunião. Além disso, são poucos os profissionais que constituem o GTEM, sendo que estes quando estão de férias ou atestado de saúde, fazem uma falta significativa na avaliação dos óbitos, não sendo facilmente substituíveis por conta da compreensão do processo. O resultado dessas situações é o acúmulo de casos e o distanciamento do prazo almejado pelo GTEM de 120 dias para finalizar a investigação e avaliação.

Para o melhor funcionamento do GTEM, algumas mudanças são projetadas, como a instituição de 60 dias para realizar todo o processo de investigação, avaliação e interlocução com as unidades, bem como a incorporação de um membro da Vigilância Sanitária, visando intervenções em situações que são de responsabilidade desta especialidade.

Em síntese, a finalidade da criação do GTEM dentro do DSN é a realização da interlocução com as unidades do distrito, visando a redução da taxa da mortalidade infantil, focando nos óbitos considerados evitáveis. Esta interlocução é a realização de uma conversa, expondo as situações levantadas e recomendações propostas, com os atores envolvidos no atendimento da mulher e do bebê. A Figura 1 representa a interlocução entre o CPOMIF e a APS, por meio do GTEM do DSN.

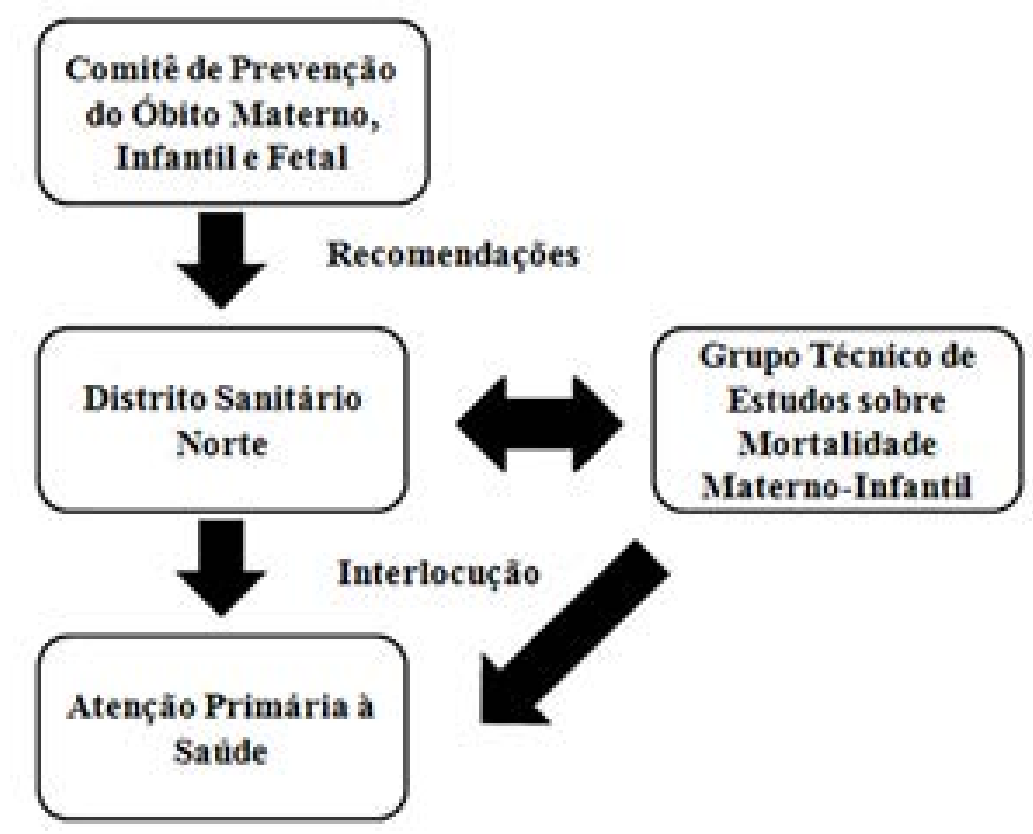

Figura 1 - Interlocução entre o CPOMIF e a APS. Florianópolis, Santa Catarina, Brasil, 2017

\section{A Interlocução com a Atenção Primária à Saúde}

Concluída a análise do óbito pelo GTEM, é realizada a interlocução com a APS, mais especificamente, com o Centro de Saúde referência do local de moradia materno. Esta conversa é efetuada pelas apoiadoras de atenção primária, que são profissionais que fornecem suporte técnico aos centros de saúde, ou, se casos mais sérios, pelos profissionais da Vigilância Epidemiológica do DSN.

O momento escolhido para a realização da interlocução é a reunião geral do Centro de Saúde, buscando o envolvimento e reflexão de todos os profissionais, os que atenderam a mulher e a criança, e as outras equipes, para que haja um crescimento conjunto.

A gente faz [a interlocução] na reunião geral de equipe porque a gente acha que esse envolvimento ajuda em uma coisa que, aquela equipe não fez, mas não tá livre de fazer. (Informante-chave) 
As apoiadoras expõem o resumo do caso, juntamente com as situações levantadas, as recomendações e a avaliação da evitabilidade proposta pelo GTEM. Após a exposição do caso, a equipe discute se concorda com os pontos levantados, colocando sugestões e complementando. Este tempo de interlocução é também aproveitado para sanar dúvidas referentes aos cuidados com a saúde materno-infantil.

Uma avaliação dos profissionais sobre o processo de interlocução apresentou resultado favorável. As equipes reagem de forma positiva à interlocução, considerando importante a discussão do caso e compreendendo que é uma forma de rever atitudes, dinâmicas e processos de trabalho que podem ser melhorados, sendo pontuado como um momento de educação continuada.

As equipes são preparadas para receber a devolutiva, de forma especial e permanente, o real objetivo da realização dessa interlocução, com ênfase na mudança de condutas, minimização das fragilidades e dos erros. Busca-se focalizar a redução da mortalidade infantil e fetal, e não a culpabilização dos atores envolvidos no óbito. Quando um assunto é mais delicado, o profissional é chamado de forma individual para conversar sobre as condutas tomadas.

Foi uma coisa que a gente trabalhou bastante, de não ir com a culpabilização, de ir que os processos de trabalho se dão porque a gente tá muito tecnicamente naquilo, porque a gente não tem tempo [...] mas que a gente sempre tem que rever os nossos processos e discutir isso nas reuniões pra evitar, minimizar essas fragilidades, esses erros, essas coisas que podem comprometer. (Informante-chave)

O tempo é uma questão importante, sendo a interlocução realizada o mais imediato possível, para que o caso não se distancie da memória dos envolvidos e mais detalhes possam ser considerados. Quando as recomendações são referentes à atenção hospitalar ou consultórios particulares, a responsabilidade da interlocução é do CPOMIF.

Como o CPOMIF não consegue realizar as avaliações em tempo real, o DSN optou por não esperar a avaliação do Comitê para realizar a interlocução com a APS. Assim, quando ocorre a avaliação pelo CPOMIF e havendo divergências nas recomendações realizadas pelo GTEM, a vigilância distrital entra em contato com o coordenador do Centro de Saúde para repassar as novas orientações, ou corrigir alguma conduta previamente orientada.

A interlocução efetua-se com o resumo do caso, as situações levantadas, as recomendações e avaliação do caso. As principais situações levantadas para o DSN, de 2014 a 2016, estavam relacionadas a: gestação não planejada; acesso facilitado ao prénatal; falta de dados no pré-natal privado; Infecção do Trato Urinário materna; obesidade; mãe tabagista; bolsa rota; trabalho de parto prematuro; doença hipertensiva específica da gravidez; vaginose não tratada; corioamnionite; multípara; falta de vínculo com a unidade; sífilis materna não tratada; falta de adesão da mulher ao pré-natal; caso social grave; HIV positivo; e falta de registro no pré-natal.

Ainda, as principais recomendações, relacionadas à APS do DSN, dos últimos três anos foram: rever planejamento de vida sexual e reprodutivo; rever rastreio de Infecção do Trato Urinário; encaminhar para aconselhamento genético; rever orientações realizadas pela ESF; realizar busca ativa; atentar para a curva de altura do fundo uterino; ensinar a posição correta para o sono do bebê, bem como fornecer instruções e material sobre broncoaspiração; sensibilizar para o tratamento do tabagismo; envolver a equipe do consultório de rua; registrar ações em prontuário; tratar parceiro com sífilis; envolver o NASF; orientar sobre a movimentação fetal e sinais de alarme e rever encaminhamento para o pré-natal de alto risco.

Dos 48 casos pertencentes ao DSN e avaliados pelo CPOMIF entre 2014 a 2016, 26 foram considerados evitáveis, sendo que 20 destes foram considerados eventos evitáveis por adequada atenção à mulher na gestação - o pré-natal. Ainda, 16 casos foram considerados inevitáveis e 6 inconclusivos. 
Com relação à taxa de mortalidade infantil, estudos têm apontado a redução das taxas referentes às cidades brasileiras nos últimos anos, como no Distrito Federal, Fortaleza e Recife ${ }^{(4-5,11)}$. Ao encontro destes dados, Florianópolis apresentou redução significativa, caindo de 9,15, em 2012, para 5,29 óbitos por mil nascidos vivos, em 2016, concordando como cenário nacional|(12).

O CPOMIF, ao realizar a vigilância dos óbitos, executa a análise dos óbitos maternos, infantis e fetais de Florianópolis. O papel desempenhado pelos comitês foi considerado relevante para a queda da mortalidade infantil. Os comitês foram apontados como espaço de educação permanente, onde se realiza a discussão sobre o processo que resultou no óbito com as equipes de saúde local|(13).

Um estudo realizado em Recife demonstrou diversas similaridades na estrutura e organização do comitê em relação ao GTEM. Assim como Florianópolis, Recife possui a divisão administrativa em Distritos Sanitários, compondo o grupo de vigilância dos óbitos os gestores, gerentes e profissionais da assistência e vigilância, indo ao encontro da realidade do DSN. Ainda, o processo de operacionalização da vigilância do óbito é equivalente ao resultado desta pesquisa, dividindo-o em constatação e identificação dos óbitos, a partir da DO, investigação epidemiológica, domiciliar, ambulatorial e hospitalar, discussão dos óbitos com os profissionais encarregados e encaminhamento das propostas para as instituições envolvidas ${ }^{(14)}$.

A ideia da criação do GTEM do DSN deu-se pelos dados do distrito em relação à mortalidade. Como apresentado nos resultados deste estudo, em Florianópolis, aproximadamente $54,1 \%(n=26)$ dos óbitos do DSN foram considerados evitáveis por adequada atenção à mulher na gestação, refletindo um potencial de redução associado à melhora da assistência pré-natal realizada no distrito. Por meio destes dados, os gestores locais propõem a criação do GTEM com o objetivo de realizar interlocução com a APS e mudar a realidade do DSN em relação à mortalidade.

Em relação à evitabilidade, estudos mostram quadro similar ao de Florianópolis, onde os resultados demonstram que as grandes maiorias dos óbitos de Montes Claros e do Distrito Federal, considerados evitáveis, possuíam o componente "reduzível por adequada atenção à gestação" mais frequente ${ }^{(4-5)}$.

Os comitês possuem caráter educativo e formativo, buscando prevenir óbitos por meio da realização de capacitações e sensibilização de profissionais e instituições. Os comitês sugerem medidas de prevenção e intervenção às instituições, visando à correção de possíveis distorções e à redução da mortalidade infanti|(8).

Os serviços de saúde possuem atuação essencial na prevenção dos óbitos evitáveis, como o que diz respeito à vigilância de óbitos infantis, uma vez que a análise desses óbitos possibilita aferir a qualidade da assistência, identificando as necessidades de intervenção, alteração e organização no processo de trabalho, buscando a melhoria do cuidado(15).

Ao realizar a interlocução com a APS, o DSN cria um meio de comunicação e revisão de condutas para melhorar a assistência prestada à saúde materno-infantil. Um estudo ressaltou a realização da interlocução com as equipes de saúde de Recife, expondo que a discussão dos óbitos com os envolvidos na assistência à mulher e aos bebês se realiza nos DS ou nos hospitais onde ocorreram os óbitos, com finalidade educativa, reflexiva e propositiva, não coercitiva ou punitiva, e enfoque na busca dos determinantes e fatores de evitabilidade, para avaliação da atenção à saúde e proposição de medidas de intervenção ${ }^{(14)}$.

Um estudo realizado em Londrina, no Paraná, mostrou que um dos principais desafios para reduzir a mortalidade infantil está relacionado à qualificação da atenção pré-natal, atenção realizada, com grandes proporções de demanda, pela APS. A ausência de registros adequados em prontuários e problemas na comunicação entre profissional e 
gestante também foram mencionados como falta de qualidade. Esses desafios evidenciam que, apesar da boa cobertura da atenção pré-natal, é preciso investir na melhoria da sua qualidade. Assim, revela-se a importância de investimentos para aprimorar a qualidade da atenção, motivar e capacitar profissionais de saúde para uma adequada adesão aos protocolos existentes e promover a articulação dos serviços de atenção básica e de assistência ao parto ${ }^{(13)}$.

Em relação ao pré-natal, outro estudo demonstra que, apesar do aumento do número de consultas no pré-natal e da cobertura, a assistência prestada não apresenta qualidade satisfatória, o que acaba por comprometer a prevenção, diagnóstico e tratamento das mães e bebês ${ }^{(16)}$.

Estes resultados refletem que, mesmo com o aumento da cobertura da Estratégia Saúde da Família e a redução na taxa de mortalidade infantil em contexto nacional, ainda há índices muito altos e incompatíveis com uma boa qualidade de vida.

\section{CONSIDERAÇÕES FINAIS}

Este estudo de caso evidenciou a estratégia organizacional do DSN ao criar o GTEM para realizar a interlocução das recomendações aos serviços de saúde. Trata-se de uma iniciativa única no município, servindo como exemplo aos demais distritos, para a efetivação da atribuição do Comitê de realizar as devolutivas às instituições de saúde envolvidas no caso de óbito.

A construção da explanação foi ao encontro das proposições iniciais deste estudo, revelando que o processo de interlocução com a APS acontece mensalmente na reunião de equipe dos centros de saúde, por meio da apresentação do caso e diálogo com a equipe. Também, o estudo amplia as proposições ao expor as formas de interlocução, por meio de reuniões, documentos e diálogos com os profissionais envolvidos. O grupo técnico, ao realizar a exposição dos dados no seminário anual realizado no DSN, promove uma interlocução indireta com as demais equipes que não possuem óbitos, sendo uma forma de educação continuada.

A interação entre Comitê e o GTEM favorece a eficácia do trabalho realizado, refletindo na qualidade do atendimento prestado e, consequentemente, na revisão e redução dos danos, diminuindo a mortalidade infantil evitável. Desta forma, este estudo contribuiu para demonstrar um exemplo positivo de gestão local, servindo de modelo para os demais distritos do município e para o sistema de saúde como um todo.

Embora este estudo limite-se a um único contexto e caso, pode-se interpretá-lo como exemplar, devido à eficácia da interlocução entre nível central e local por meio de um órgão estruturado. Estudos com este tema poderiam ser desenvolvidos em outros contextos e casos, buscando compreender as diferentes formas de trabalho, agregando e compartilhando as experiências, com vistas à melhoria da assistência e gestão da saúde materno-infantil.

\section{REFERÊNCIAS}

1. Gaiva MAM, Fujimori E, Sato APS. Maternal and child risk factors associated with neonatal mortality. Texto contexto- enferm [Internet]. 2016 [acesso em 21 abr 2017]; 25(4). Disponível em: http://dx.doi. org/10.1590/0104-07072016002290015.

2. United Nations (UN). Sustainable development knowledge platform. [Internet]. 2015 [acesso em $25 \mathrm{abr}$ 2016]. Disponível em: https://sustainabledevelopment.un.org/.

3. Silva VLS, Santos IS, Medronha NS, Matijasevich A. Mortalidade infantil na cidade de Pelotas, estado 
do Rio Grande do Sul, Brasil, no período 2005-2008: uso da investigação de óbitos na análise das causas evitáveis. Epidemiol. Serv. Saúde [Internet]. 2012 [acesso em 24 mar 2017]; 21(2). Disponível em: http:// dx.doi.org/10.5123/S1679-49742012000200009.

4. Oliveira E, Souto MB, Santos RO, Fonseca RL, Matos FV, Caldeira AP. Mortalidade infantil evitável em Montes Claros, Minas Gerais, Brasil, 1999-2011. Rev. baiana saúde pública [Internet]. 2015 [acesso em 25 jun 2017]; 39(2):397-407. Disponível em: http://rbsp.sesab.ba.gov.br/index.php/rbsp/article/ view/1688/1245.

5. Canabrava PBE, Rocha JLFN, Costa AM, Elias KJ, Lima RV. Mortalidade infantil por causas evitáveis no Distrito Federal no período de 2003 a 2012. Rev Med Saúde Brasília [Internet]. 2016 [acesso em 21 mar 2017]; 5(2). Disponível: https://portalrevistas.ucb.br/index.php/rmsbr/article/download/7000/4564.

6. Oliveira CM, Bonfim CV, Guimarães MJB, Frias PG, Medeiros ZM. Infant mortality: temporal trend and contribution of death surveillance. Acta Paul. Enferm. [Internet]. 2016 [acesso em 23 mar 2017]; 29(3). Disponível: http://dx.doi.org/10.1590/1982-0194201600040.

7. Figueiredo PP, Lunardi Filho WD, Lunardi VL, Pimpão FD. Mortalidade infantil e pré-natal: contribuições da clínica à luz de Canguilhem e Foucault. Rev. Latino-Am. Enfermagem [Internet]. 2012 [acesso em 28 nov 2017]; 20(1). Disponível em: http://dx.doi.org/10.1590/S0104-11692012000100026.

8. Ruoff $A B$, Andrade SR, Schmitt MD. Atividades desenvolvidas pelos comitês de prevenção do óbito infantil e fetal: revisão integrativa. Rev. Gaúcha Enferm. [Internet]. 2017 [acesso em 01 out 2017]; 38(1). Disponível em: http://dx.doi.org/10.1590/1983-1447.2017.01.67342.

9. Ministério da Saúde (BR). Secretaria de Vigilância em Saúde, Secretaria de Atenção à Saúde. Manual de vigilância do óbito infantil e fetal e do comitê de prevenção do óbito infantil e fetal. 2. ed. Brasília: Ministério da Saúde; 2009. 96 p.

10. Yin RK. Estudo de caso: planejamento e métodos. 4.ed. Porto Alegre: Bookman; 2010.

11. Sanders LSC, Pinto FJM, Medeiros CRB, Sampaio RMM, Viana RAV, Lima KJ. Mortalidade infantil: análise de fatores associados em uma capital do Nordeste brasileiro. Cad. saúde colet. [Internet]. 2017 [acesso em 17 abr 2017]; 25(1). Disponível em: http://dx.doi.org/10.1590/1414-462x201700010284.

12. Ministério da Saúde (BR). Departamento de Informática do SUS (DATASUS), Sistema de Informações sobre Mortalidade - Dezembro de 2016. Painel de Monitoramento da Mortalidade Infantil e Fetal. Brasília: Ministério da Saúde; 2016.

13. Pizzo LGP, Andrade SM, Silva AMR, Melchior R, González AD. Mortalidade infantil na percepção de gestores e profissionais de saúde: determinantes do seu declínio e desafios atuais em município do sul do Brasil. Saúde Soc. [Internet]. 2014 [acesso em 26 mar 2017]; 23(3):908-918. Disponível em: http://www. scielo.br/pdf/sausoc/v23n3/0104-1290-sausoc-23-3-0908.pdf.

14. Oliveira CM, Bonfim CV, Guimarães MJB, Frias PG, Antonino VCS, Medeiros ZM. Infant mortality surveillance in Recife, Pernambuco, Brazil: operationalization, strengths and limitations. Epidemiol. serv. saúde. [Internet]. 2017 [acesso em 15 maio 2017]; 26(2). Disponível em: http://dx.doi.org/10.5123/s167949742017000200019.

15. Santana M, Aquino R, Medina MG. Efeito da Estratégia Saúde da Família na vigilância de óbitos infantis. Rev. Saúde Públ. [Internet]. 2012 [acesso em 17 maio 2017]; 46(1). Disponível em: http://dx.doi. org/10.1590/S0034-89102011005000081.

16. Silva ALA, Mendes ACG, Miranda GMD, Santos Neto PM. Assistência ao parto no Brasil: uma situação crítica ainda não superada. Rev. Bras. Saúde Matern. Infant. [Internet]. 2016 [acesso em 25 abr 2017]; 16(2). Disponível: http://dx.doi.org/10.1590/1806-93042016000200004. 
Recebido: 01/12/2017

Finalizado: 08/03/2019

Autor Correspondente:

Alexandra Ferreira

Universidade Federal de Santa Catarina

Servidão Silvino Izidoro Pires, 88 - 88066-345 - Florianópolis, SC, Brasil

E-mail: xanferr@gmail.com

\section{Contribuição dos autores:}

Contribuições substanciais para a concepção ou desenho do estudo; ou a aquisição, análise ou interpretação de dados do estudo - AF, SRA, ABR, ACAX

Aprovação da versão final do estudo a ser publicado - LCFB 